\title{
Correction: Some new theorems of expanding mappings without continuity in cone metric spaces
}

Shaoyuan $\mathrm{Xu}^{1 *}$, Suyu Cheng ${ }^{2}$ and Yan $\mathrm{Han}^{3}$

\section{"Correspondence:}

xushaoyuan@126.com

${ }^{1}$ Department of Mathematics and

Statistics, Hanshan Normal

University, Chaozhou, 521041, China

Full list of author information is

available at the end of the article

\begin{abstract}
In this note we correct some errors that appeared in the article (Han and Xu in Fixed Point Theory Appl. 2013:3, 2013) by modifying some conditions in the main theorems and corresponding corollaries.
\end{abstract}

MSC: $47 \mathrm{H} 10 ; 54 \mathrm{H} 25$

Keywords: cone metric space; expanding mapping; common fixed point

\section{Correction}

Upon critical examination of the main results and their proofs in [1], we note several critical errors in the conditions of the main theorems in [1]. These errors lead to subsequent errors in the corresponding corollaries in [1].

In this note, we would like to supplement several conditions, which are used in their proofs but not referred to in the conditions of the main results, to achieve our claim.

The following theorem is a modification to [1, Theorem 2.1]. The proof is the same as that in [1]. We will attain the desired goal by adding two conditions to that in [1, Theorem 2.1]. We state that Theorem 2.1 in [1] is replaced by the following theorem.

Theorem 2.1 Let $(X, d)$ be a complete cone metric space. Suppose that the mapping $f$ : $X \rightarrow X$ is onto and such that

$$
d(f x, f y) \geq a_{1} d(x, y)+a_{2} d(x, f x)+a_{3} d(y, f y)+a_{4} d(x, f y)+a_{5} d(y, f x),
$$

for all $x, y \in X$, where $a_{i}(i=1,2,3,4,5)$ satisfy $a_{2} \geq 0, a_{4} \geq 0, a_{1}+a_{2}+a_{3}>1$ and $a_{3} \leq 1+a_{4}$. Then $f$ has a fixed point.

Remark 2.1 Compared to Theorem 2.1 in [1], Theorem 2.1 mentioned above possesses the conditions $a_{2} \geq 0$ and $a_{4} \geq 0$ while, unluckily, Theorem 2.1 in [1] does not. The reason for supplementing these conditions is the fact that in the proof of [1, Theorem 2.1] we have used the conditions $a_{2} \geq 0$ and $a_{4} \geq 0$ to ensure that the two deductions

(i) $d\left(x_{n+1}, x_{n-1}\right) \geq d\left(x_{n+1}, x_{n}\right)-d\left(x_{n-1}, x_{n}\right)$ implies $a_{4} d\left(x_{n+1}, x_{n-1}\right) \geq a_{4} d\left(x_{n+1}, x_{n}\right)-a_{4} d\left(x_{n-1}, x_{n}\right)$

and

( 2014 Xu et al.: licensee Springer. This is an Open Access article distributed under the terms of the Creative Commons Attribution License (http://creativecommons.org/licenses/by/2.0), which permits unrestricted use, distribution, and reproduction in any medium, provided the original work is properly cited. 
(ii) $d(p, q) \geq d\left(p, x_{n+1}\right)-d\left(q, x_{n-1}\right)$ implies $a_{2} d(p, q) \geq a_{2} d\left(p, x_{n+1}\right)-a_{2} d\left(q, x_{n-1}\right)$ must be valid.

Similarly, the following theorem is a modification to [1, Theorem 2.5]. The proof is the same as that in [1]. We state that Theorem 2.5 in [1] is replaced by the following theorem.

Theorem 2.5 Let $(X, d)$ be a complete cone metric space. Suppose the mappings $f, g: X \rightarrow$ $X$ are onto and satisfy

$$
d(f x, g y) \geq a_{1} d(x, y)+a_{2} d(x, f x)+a_{3} d(y, g y)+a_{4} d(x, g y)+a_{5} d(y, f x),
$$

for all $x, y \in X$, where $a_{i}(i=1,2,3,4,5)$ satisfy $a_{3} \geq 0, a_{5} \geq 0, a_{1}+a_{2}+a_{3}>1$ and $a_{2} \leq 1+a_{5}$, $a_{3} \leq 1+a_{4}$. Then $f$ and $g$ have a common fixed point.

Accordingly, the following two corollaries are modifications to Corollary 2.2 and Corollary 2.6 in [1], and we state that the latter corollaries are replaced by the former ones, respectively.

Corollary 2.2 Let $(X, d)$ be a complete cone metric space. Suppose the mapping $f: X \rightarrow X$ is onto and such that

$$
d(f x, f y) \geq k d(x, y)+l d(x, f x)+p d(y, f y),
$$

for all $x, y \in X$, where $p \leq 1, l \geq 0$ and $k+l+p>1$. Then $f$ has a fixed point.

Corollary 2.6 Let $(X, d)$ be a complete cone metric space. Suppose the mappings $f, g: X \rightarrow$ $X$ are onto and such that

$$
d(f x, g y) \geq \alpha d(x, y)+\beta[d(x, f x)+d(y, g y)]+\gamma[d(x, g y)+d(y, f x)]
$$

for all $x, y \in X$, where $\beta \geq 0, \gamma \geq 0, \beta \leq 1+\gamma$ and $\alpha+2 \beta>1$. Then $f$ and $g$ have a common fixed point.

\section{Author details}

${ }^{1}$ Department of Mathematics and Statistics, Hanshan Normal University, Chaozhou, 521041, China. ${ }^{2}$ Library, Hanshan Normal University, Chaozhou, 521041, China. ${ }^{3}$ Department of Mathematics, Zhaotong University, Zhaotong, 657000, China

\section{Acknowledgements}

The research is partially supported by the PhD Start-up Fund of Hanshan Normal University, Guangdong Province, China (No. QD20110920).

Received: 28 July 2014 Accepted: 28 July 2014 Published: 19 August 2014

\section{References}

1. Han, $Y, X u, S$ : Some new theorems of expanding mappings without continuity in cone metric spaces. Fixed Point Theory Appl. 2013, 3 (2013) 